\title{
Acute Leukemia of Ambiguous Lineage
}

National Cancer Institute

\section{Source}

National Cancer Institute. Acute Leukemia of Ambiguous Lineage. NCI Thesaurus. Code C7464.

An acute leukemia in which the blasts lack sufficient evidence to classify as myeloid or lymphoid or they have morphologic and/or immunophenotypic characteristics of both myeloid and lymphoid cells. (WHO, 2001) 